\title{
Multi-Fluid Flows with Weak and Strong Discontinuous Interfaces Using an Elemental Enriched Space
}

\author{
Sergio R. Idelsohn ${ }^{\mathrm{a}, \mathrm{b}, \mathrm{c}, \mathrm{d}, *}$, Juan M. Gimenez ${ }^{\mathrm{c}, \mathrm{d}}$, Norberto M. Nigro ${ }^{\mathrm{c}, \mathrm{d}}$ \\ ${ }^{a}$ Institució Catalana de Recerca i Estudis Avançats (ICREA), Barcelona, Spain \\ ${ }^{b}$ Centre Internacional de Mètodes Numèrics en Enginyeria (CIMNE), Barcelona, Spain \\ ${ }^{c}$ Centro de Investigación de Métodos Computacionales (CIMEC-UNL-CONICET), Santa \\ Fe, Argentina \\ ${ }^{d}$ Facultad de Ingeniería y Ciencias Hídricas, Universidad Nacional del Litoral, Santa Fe, \\ Argentina
}

\begin{abstract}
In a previous paper the authors present an elemental enriched space to be used in a finite element framework (EFEM) capable to reproduce kinks and jumps in an unknown function using a fixed mesh in which the jumps and kinks do not coincide with the inter-element boundaries. In this previous publication, only scalar transport problems where solved (thermal problems). In the present work these ideas are generalized to vectorial unknowns, in particular the incompressible Navier-Stokes equations for multi-fluid flows presenting internal moving interfaces. The advantage of the EFEM compared with the global enrichment is the important reduction of the computing time when the internal interface is moving. In the EFEM the matrix to be solved at each time-step has, not only the same amount of degrees of freedom (DOFs) but also has always the same connectivity between the DOFs. This frozen matrix-graph improves enormously the efficiency of the solver. Another characteristic of the elemental enriched space presented here is that allows a linear variation of the jump, improving the convergence rate compared with other enriched spaces that have a constant variation of the jump. Furthermore, the implementation in any existing finite element code is extremely easy with the version presented here because the new shape functions are based on the usual FEM shape functions for triangles or tetrahedrals and, once
\end{abstract}

${ }^{*}$ Corresponding author: Sergio R. Idelsohn; Postal Address; Gran Capitan s/n; Edificio C1, Campus Nord UPC; 08034 Barcelona, Spain; Tel.: +34 93401 1829; E-mail: sergio@cimne.upc.edu 
statically condensed the internal DOFs, the resulting elements have exactly the same number of unknowns as the non-enriched finite elements.

Keywords: Enriched FE spaces, Internal interfaces, Discontinuous fields, Multi-fluids, EFEM, Navier-Stokes equations, CFD, Incompressible fluid flows.

\section{Introduction}

In a previous paper [1] the authors presented an elemental enriched space capable to reproduce kinks and jumps of the unknown functions using a fixed mesh in which the jumps and kinks do not coincide with the inter-element boundaries. In that publication, only thermal problems where solved in which the unknown variable was a scalar function. In this work we generalize the previous ideas to a vectorial field like the incompressible Navier-Stokes equations.

Kinks and jumps in the velocity and in the pressure fields are present in many engineering problems, in particular in multi-fluids and fluid-structure interaction problems. In the case of multi-fluids (several fluids with different physical properties), the dynamics of the interface between the fluids involved plays a dominant role. The computation of the interface between various immiscible fluids or the free surfaces is extremely difficult because neither the shape nor the positions of the interfaces are a priori known. The approaches to solve these problems are mainly two: one is based on using a moving mesh that follows the discontinuity, named interface-tracking methods, and the second based on using a fixed mesh (some times refined in that part of the domain where the interface cross during the evaluation) named interfacecapturing methods.

In this last method (see $[2,3,4]$ ), the interface is determined by an implicit function immersed in an Eulerian (fix) mesh and he flow problem is solved considering the fluids as a single effective fluid with variable properties. Popular methods of this type are the Volume-of-Fluid (VOF) technique (see $[5,6,7]$ ) the Level-Set method (see, for instance $[8,9,10,11]$ ) which advect the interface using Eulerian strategies, and the Particle Finite Element Method Second Generation (PFEM-2) [12, 13, 14, 15] which employs a Lagrangian method.

Independently of the method used to move the internal interface, the problem in fixed mesh methods is that the change of physical material prop- 
erties along the interface introduces kinks or possibly jumps which must be captured in the solution of the global problem in order to have accurate results. Some authors try to fulfill this issue refining the mesh near the interface without introducing any possibility to have a kink or a jump inside the elements.

For such problems when the interface does not necessarily conform to the element edges (in 2D) or faces (in 3D), the finite element solution, either for continuous or discontinuous approximations across inter-element boundaries suffers of sub-optimal convergence rate. This poor approximation leads to spurious velocities near the interface that may significantly affect the precision and the robustness of numerical simulations (see e.g., [16]). Furthermore, the need of a local refinement around the interface, implies the refinement of the mesh in almost the entire domain where the possible position of the interface may go through when the interface move.

A number of methods have been developed to overcome these difficulties. One possibility is to add degrees of freedom or enrich the finite element space at the elements cut by the interface. Minev et al. [17], and later Chessa and Belytschko [18], adopted an enrichment technique nowadays called XFEM, a name coined in the context of fracture mechanics or named also GFEM by other authors [19]. Both approaches lead to optimal orders of convergence, but the main drawback is that the additional degrees of freedom cannot be eliminated before assembly. The XFEM approach has also been used recently in two-phase flows [20,21]. These kinds of enrichment have been also called global enrichment or nodal enrichment, or as stated before XFEM or GFEM. A method that avoid the inclusion of additional degrees of freedom is one that allow to statically condense the additional degree of freedoms prior to the assembly. For this reason, these kinds of enrichment have been named elemental enrichment or $\operatorname{EFEM}[22,23]$. A generalization of the treatment of kinks and jumps in the pressure field was presented by Ausas et al. in [24]. However, the enriched space proposed in [24] works satisfactorily for the pressure field in the Navier-Stokes equations but does not work correctly for the enrichment of the temperature field in a typical thermal problem or for the enrichment of the displacement or the velocity field in solid or fluid mechanics problems. As previously stated, Idelsohn et al. presented in[1] a new elemental enriched space that allow a better approximation for second order equations in which an integration by parts is needed. The generalization of these ideas to the incompressible Navier-Stokes equations are presented next. 
The advantage of the EFEM compared with the global enrichment is the important reduction of the computing time when the internal interface is moving. In the EFEM the matrix to be solved at each time-step has, not only the same amount of degrees of freedom (DOFs) but also has always the same connectivity between the DOFs. This means that the matrix-graph remains constant while in the XFEM the matrix-graph is permanently changing. This frozen graph improves enormously the efficiency of the solver, mainly in 3D problems $[22,23]$. The disadvantage of the EFEM is the impossibility to be exactly consistent with the internal continuities required for the variational form. The way to mitigate these inconsistencies for the case of multi-fluids (also called variational crimes) is one of the main targets of this work.

Another characteristic of the elemental enriched space presented here is that allows a linear variation of the jump, improving the convergence rate to the exact solution compared with other enriched spaces that have a constant variation of the jump. The implementation in any existing finite element code is extremely easy in both: two and three spatial dimensions. This is because the new shape functions are based on the usual FEM shape functions for triangles or tetrahedrals and, once statically condensed the internal DOFs, the resulting elements have exactly the same number of unknowns as the non-enriched FE. To show the accuracy of the new space proposed, simple but very convincing examples of the solution of the Navier-Stokes equations for single phase and multi-fluid flows using a fixed background mesh are presented as numerical examples.

\section{The governing equations}

Conservation of linear momentum

The momentum conservation in the entire domain reads

$$
\rho \frac{D \mathbf{u}}{D t}=\nabla \cdot \sigma+\mathbf{b}
$$

where $\rho$ is density, $\mathbf{u}$ the velocity vector, $\sigma$ the stress tensor, $\mathbf{b}$ a source vector and $\frac{D \mathbf{u}}{D t}$ the material derivative is the acceleration vector that can be also written in an Eulerian frame as $\frac{D \mathbf{u}}{D t}=\frac{\partial \mathbf{u}}{\partial t}+\mathbf{u} \cdot \nabla \mathbf{u}$.

For the incompressible Navier-Stokes equations the stress are related to the velocity gradients and the pressure through

$$
\sigma=2 \mu \nabla^{s} \mathbf{u}-p \mathbf{I}
$$


where $\mu$ is the dynamic viscosity, $p$ the pressure, $\mathbf{I}$ the identity matrix and $\nabla^{s} \mathbf{u}$ is the symmetric gradient tensor of the velocity field.

Possible boundary conditions on the boundary domains are

$$
\left\{\begin{array}{l}
\bar{\sigma}_{n}=\sigma_{n}=\left(2 \mu \nabla^{s} \mathbf{u}-p \mathbf{I}\right) \cdot \mathbf{n} \quad \text { on } \Gamma_{\sigma} \\
\overline{\mathbf{u}}=\mathbf{u} \quad \text { on } \Gamma_{u}
\end{array}\right.
$$

where $\bar{\sigma}_{n}$ and $\overline{\mathbf{u}}$ represent known external values and $\mathbf{n}$ the outside normal vector.

Possible internal conditions at the internal interface are

$$
\sigma_{n}^{+}=\sigma_{n}^{-} \quad \text { on } \Gamma_{\text {int }}
$$

where $\sigma_{n}^{+}$and $\sigma_{n}^{-}$represent the normal stresses on both side of the interface considering positive in the sense of the outside unit normal to the interface respectively.

\section{Conservation of mass}

Mass conservation, or the continuity equation, must be satisfied in the entire fluid domain. Assuming incompressible fluid flow, continuity requires the divergence of the velocity to be zero

$$
\nabla \cdot \mathbf{u}=0
$$

with boundary conditions

$$
\mathbf{u}_{n}=\mathbf{u} \cdot \mathbf{n}=\overline{\mathbf{u}}_{n} \quad \text { on } \Gamma_{u}
$$

On the internal interfaces the incompressible condition forces to have

$$
\mathbf{u}_{n}^{+}=\mathbf{u}_{n}^{-} \quad \text { on } \Gamma_{\text {int }}
$$

where again, $\mathbf{u}_{n}^{+}$and $\mathbf{u}_{n}^{-}$represent the normal velocity on both side of the interface.

\section{The Finite Element discretization and the enriched space}

\subsection{Conservation of linear momentum}

In a Finite Element approximation, artificial kinks of the unknown functions between two neighboring elements are introduced. In this case the following constrain must be added

$$
\sigma_{n}^{\text {ele }}=\sigma_{n}^{\text {neigh }} \quad \text { on } \Gamma_{l}
$$


where $\Gamma_{l}$ represents all the finite element boundaries, see Figure $1, \sigma_{n}^{\text {ele }}$ the normal stresses at the finite element boundaries and $\sigma_{n}^{\text {neigh }}$ the normal stresses on the finite element boundaries of the neighboring elements.

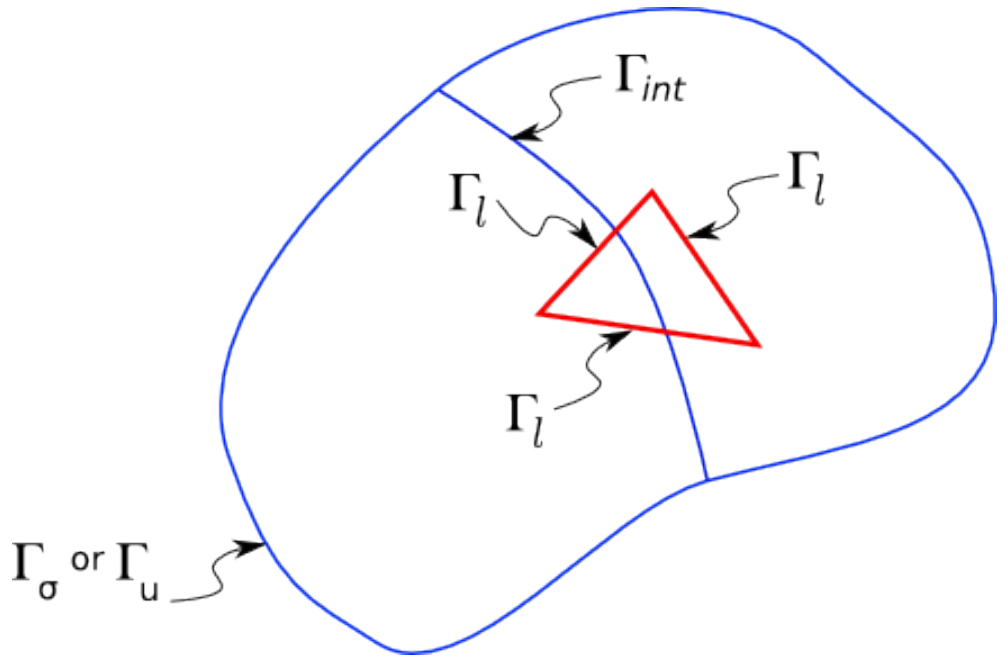

Figure 1: Different interface names

The weighted residual form of the previous equation is

$$
\begin{array}{r}
\sum_{\Omega_{l=1}}^{\Omega_{l=N e}} \int_{\Omega_{l}} \mathbf{w} \cdot\left(\rho \frac{D \mathbf{u}}{D t}-\nabla \cdot 2 \mu \nabla^{s} \mathbf{u}+\nabla p-\mathbf{b}\right) d \Omega-\int_{\Gamma_{\sigma}} \mathbf{w} \cdot\left(\overline{\sigma_{n}}-\sigma_{n}\right) d \Gamma- \\
-\int_{\Gamma_{\text {int }}} \mathbf{w} \cdot\left(\sigma_{n}^{+}-\sigma_{n}^{-}\right) d \Gamma-\int_{\Gamma_{l}} \mathbf{w} \cdot\left(\sigma_{n}^{\text {ele }}-\sigma_{n}^{\text {neigh }}\right) d \Gamma=0
\end{array}
$$

where $\mathbf{w}$ is the vector of weighting functions (equal to the shape function to be used to approximate the velocity field in the case of Galerkin approximations).

Remark: For an Eulerian time integration, the weighting function should be modified in order to get spatial stabilized schemes[25].

After the integration by parts, (9) remains:

$$
\sum_{\Omega_{l=1}}^{\Omega_{l=N e}}\left[\int_{\Omega_{l}} \mathbf{w} \cdot\left(\rho \frac{D \mathbf{u}}{D t}-\mathbf{b}\right)+\nabla \mathbf{w}::\left(\mu \nabla^{s} \mathbf{u}+p \mathbf{I}\right)\right] d \Omega-\int_{\Gamma_{\sigma}} \mathbf{w} \cdot \overline{\sigma_{n}} d \Gamma
$$


Note that due to the continuity of the weighting functions $\mathbf{w}$, after integration by parts, all the integral on the internal interfaces at the real interface $\Gamma_{\text {int }}$ as well as on the artificial inter-element interfaces $\Gamma_{l}$ disappear.

Nevertheless, in order to allow the possibility to have discontinuities in the velocity field, special discontinuous shape functions will be added to the continuous standard FE shape functions. In the same way, a continuous part plus a discontinuous one will form the weighting functions. Calling $\mathbf{w}_{e}$ the discontinuous weighting function to be introduced for the enriched space and remaining the notation of $\mathbf{w}$ for the standard continuous finite element weighting functions, the integration by part will read:

$$
\begin{array}{r}
\sum_{\Omega_{l=1}}^{\Omega_{l=N e}} \int_{\Omega_{l}}\left[\mathbf{w} \cdot\left(\rho \frac{D \mathbf{u}}{D t}-\mathbf{b}\right)+\nabla \mathbf{w}::\left(\mu \nabla^{s} \mathbf{u}+p \mathbf{I}\right)\right] d \Omega-\int_{\Gamma_{\sigma}} \mathbf{w} \cdot \overline{\sigma_{n}} d \Gamma=0 \\
\sum_{\Omega_{l=1}}^{\Omega_{l=N e}} \int_{\Omega_{l}}\left[\mathbf{w}_{e} \cdot\left(\rho \frac{D \mathbf{u}}{D t}-\mathbf{b}\right)+\nabla \mathbf{w}_{e}::\left(\mu \nabla^{s} \mathbf{u}+p \mathbf{I}\right)\right] d \Omega-\int_{\Gamma_{\sigma}} \mathbf{w}_{e} \cdot \overline{\sigma_{n}} d \Gamma \ldots \\
\ldots-\int_{\Gamma_{\mathrm{int}}}\left(\mathbf{w}_{e}^{+} \cdot \sigma_{n}^{-}+\mathbf{w}_{e}^{-} \cdot \sigma_{n}^{+}\right) d \Gamma-\int_{\Gamma_{l}} \mathbf{w}_{e} \cdot \sigma_{n}^{\text {neigh }} d \Gamma=0
\end{array}
$$

The first line in (11) is the standard variational form for the continuous weighting functions $\mathbf{w}$ while the second line is the variational form for discontinuous weighting functions.

The term in $\sigma_{n}^{\text {neigh }}$ represents the normal stress to the finite element boundaries of the neighboring elements and $\sigma_{n}^{+}, \mathbf{w}_{e}^{+}, \sigma_{n}^{-}$and $\mathbf{w}_{e}^{-}$the normal stress and the enriched weighting function on both side of the internal interface. The evaluation of these terms will be discussed later.

\subsection{Conservation of mass}

As stated before, the elemental enriched space may introduce discontinuities between the two neighboring elements as well in the velocity field as in the pressure field. Furthermore, for a Galerkin approximation the corresponding weighting functions have exactly the same possible discontinuities that must be taken into account in order to satisfy the incompressible condition.

Possible velocity discontinuities between elements force to impose the following constrain

$$
\mathbf{u}_{n}^{\text {ele }}=\mathbf{u}_{n}^{\text {neigh }} \quad \text { on } \Gamma_{l}
$$


where $\mathbf{u}_{n}^{\text {ele }}$ represents the normal velocity to a boundary element and $\mathbf{u}_{n}^{\text {neigh }}$ the normal velocity on the boundary of the neighboring element.

The weighted residual form of the mass conservation with possible discontinuous velocity fields (supposing that the boundary constraint $\mathbf{u}_{n}=\overline{\mathbf{u}}_{n}$ is a priori satisfied) reads

$$
\begin{array}{r}
\sum_{\Omega_{l=1}}^{\Omega_{l=N e}} \int_{\Omega_{l}} w^{p} \nabla \cdot \mathbf{u} d \Omega-\int_{\Omega_{l}} \nabla w^{p} \cdot \mathbf{u}_{e} d \Omega+\int_{\Gamma_{\mathbf{u}}} w^{p} \overline{\mathbf{u}}_{n e} d \Gamma \\
+\int_{\Gamma_{\mathrm{int}}} w^{p}\left(\mathbf{u}_{n e}^{+}-\mathbf{u}_{n e}^{-}\right) d \Gamma+\int_{\Gamma_{l}} w^{p}\left(\mathbf{u}_{n e}^{\text {neigh }}-\mathbf{u}_{n e}^{\text {ele }}\right) d \Gamma=0
\end{array}
$$

where $w^{p}$ is the weighting function equal to the shape function to be used to approximate the pressure field in the case of Galerkin approximations and $\overline{\mathbf{u}}_{n e}$ is the enriched velocity at the boundary.

Remark: Equal order interpolation for velocity-pressure are stabilized through SUPG-PSPG in this context.

For the case of continuous velocity fields, all the boundary integrals in the previous equation disappear, but for discontinuous velocities, special care must be taken on the internal interfaces and on the boundaries between two neighboring elements crossed by the interface.

Furthermore, the pressure (and then the weighting functions $w^{p}$ ) will be also enriched with discontinuous functions. Calling $w^{p}$ the continuous weighting functions for the incompressible terms and $\mathbf{u}_{e}, w_{e}^{p}$ the enriched velocity shape functions and the pressure weighting functions respectively, four cases must be taken in consideration.

1. Continuous weighting functions and continuous velocity shape functions In this case, the weighted residual form for the incompressible equation reduces to

$$
\sum_{\Omega_{l=1}}^{\Omega_{l=N e}}\left[\int_{\Omega_{l}} w^{p} \nabla \cdot \mathbf{u} d \Omega\right]=0
$$

2. Continuous pressure weighting functions and discontinuous velocity shape functions In this case integrating by parts the divergence term, all the boundary integral terms disappear remaining

$$
-\sum_{\Omega_{l=1}}^{\Omega_{l=N e}}\left[\int_{\Omega_{l}} \nabla w^{p} \cdot \mathbf{u}_{e} d \Omega\right]+\int_{\Gamma} w^{p} \cdot \mathbf{u}_{n e} d \Gamma=0
$$


This means that the terms involved in continuous pressure weighting functions and discontinuous velocity shape functions are easily solved integrating by parts the divergence velocity term. In contrast, an integral term must be added in the whole external contour of the domain.

3. Discontinuous weighting functions and continuous velocity shape functions In this case again, (13) reduce to

$$
\sum_{\Omega_{l=1}}^{\Omega_{l=N e}}\left[\int_{\Omega_{l}} w_{e}^{p} \nabla \cdot \mathbf{u} d \Omega\right]=0
$$

4. Discontinuous weighting functions and discontinuous velocity shape functions In this case the Equation (13) remains with all the terms. An integration by parts avoid some terms on the boundary integrals, but the terms concerning the neighboring elements remain in the equation which make impossible to be exactly solved with an elemental enrichment strategy. The approximation of both neighboring terms: $\sigma_{n}^{\text {neigh }}$ in (11) and $\mathbf{u}_{n}^{\text {neigh }}$ in (13) will be discussed next.

\section{Evaluation of the jump condition for the internal interfaces}

Equation (11) introduces integral terms with the normal stresses $\sigma_{n}^{+}$and $\sigma_{n}^{-}$which represent the normal stresses on both side of the internal interface in which a jump or a kink might be located. To evaluate these terms a regularization zone on a very thin band with thickness $\epsilon$ will be considered. On this band the stress tensor will be described in local coordinates on the interface in its normal and tangent directions $n, \tau_{1}$ and $\tau_{2}$ respectively, which will be named $\sigma^{R}$. In the same way the normal unit vector in this particular coordinates will be called $\mathbf{n}^{R}$ with $\left(\mathbf{n}^{R}\right)^{T}=(1,0,0)$. For instance, for the two-dimensional case, the matrix $\sigma^{R}$ remains:

$$
\sigma^{R}=2 \mu^{*}\left[\begin{array}{cc}
\frac{\partial \mathbf{u}_{n}}{\partial n} & \frac{1}{2}\left(\frac{\partial \mathbf{u}_{\tau}}{\partial n}+\frac{\partial \mathbf{u}_{n}}{\partial \tau}\right) \\
\frac{1}{2}\left(\frac{\partial \mathbf{u}_{\tau}}{\partial n}+\frac{\partial \mathbf{u}_{n}}{\partial \tau}\right) & \frac{\partial \mathbf{u}_{\tau}}{\partial \tau}
\end{array}\right]-p \mathbf{I}
$$

The coefficient $\mu^{*}$ is a fictitious viscosity of the regularization zone. It can be considered as an orthotropic material

$$
\mu^{*}=\left[\begin{array}{cc}
\mu_{n}^{*} & 0 \\
0 & \mu_{\tau}^{*}
\end{array}\right]
$$


The tensor stress normal to the interface in the regularized region becomes

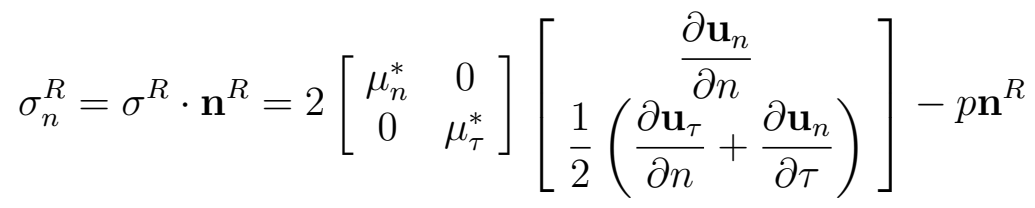

Furthermore, in this region, the derivatives in the direction of the normal to the interface may be written as (20)

$$
\frac{\partial \mathbf{u}_{n}^{R}}{\partial n}=\frac{\left\|\mathbf{u}_{n}\right\|^{+}}{\epsilon} \quad \text { and } \quad \frac{\partial \mathbf{u}_{\tau}^{R}}{\partial n}=\frac{\left\|\mathbf{u}_{\tau}\right\|^{+}}{\epsilon}
$$

where $\left\|\mathbf{u}_{\alpha}\right\|^{+}=\mathbf{u}_{\alpha}^{+}-\mathbf{u}_{\alpha}^{-}$and $\left\|\mathbf{u}_{\alpha}\right\|^{-}=\mathbf{u}_{\alpha}^{-}-\mathbf{u}_{\alpha}^{+}$represent the jump at the interface of the $\alpha$ component of the velocity including the sign.

For finite value of the jumps, this derivative tends to infinite when $\epsilon$ tends to zero. This means that the other derivatives may be neglected on this regularized region

$$
\begin{aligned}
\sigma_{n}^{R+} & =2\left[\begin{array}{cc}
\mu_{n}^{*} & 0 \\
0 & \mu_{\tau}^{*}
\end{array}\right]\left[\begin{array}{c}
\frac{\left\|\mathbf{u}_{n}\right\|^{+}}{\epsilon} \\
\frac{\left\|\mathbf{u}_{\tau}\right\|^{+}}{2 \epsilon}
\end{array}\right]-p^{+} \mathbf{n}^{R}= \\
& =2\left[\begin{array}{cc}
\frac{\mu_{n}^{*}}{\epsilon} & 0 \\
0 & \frac{\mu_{\tau}^{*}}{2 \epsilon}
\end{array}\right]\left\|\mathbf{u}^{R}\right\|^{+}-p^{+} \mathbf{n}^{R}=\mathbf{J}^{r}\left\|\mathbf{u}^{R}\right\|^{+}-p^{+} \mathbf{n}^{R}
\end{aligned}
$$

where the orthotropic coefficient matrix $\mathbf{J}^{R}$ is

$$
\mathbf{J}^{R}=\left[\begin{array}{cc}
J_{n} & 0 \\
0 & J_{\tau}
\end{array}\right]=\left[\begin{array}{cc}
\frac{2 \mu_{n}^{*}}{\epsilon} & 0 \\
0 & \frac{\mu_{\tau}^{*}}{\epsilon}
\end{array}\right]
$$

Taking into account all the previously considerations, the normal stress at the interface $\sigma_{n}^{+}$must be evaluated as

$\sigma_{n}^{+}=\sigma_{n} \cdot \mathbf{n}^{+}=\mathbf{R}^{T} \sigma^{R} \mathbf{R R}^{T} \cdot \mathbf{n}^{R}=\mathbf{R}^{T} \sigma^{R} \cdot \mathbf{n}^{R}=\mathbf{R}^{T} \sigma_{n}^{R}=\mathbf{R}\left(\mathbf{J}^{R}\left\|\mathbf{u}^{R}\right\|^{+}-p^{+} \mathbf{n}^{R}\right)$

or

$$
\sigma_{n}^{+}=\mathbf{R}^{T} \mathbf{J}^{R} \mathbf{R}\|\mathbf{u}\|^{+}-p^{+} \mathbf{R}^{T} \cdot \mathbf{n}^{R}=\mathbf{J}\|\mathbf{u}\|^{+}-p^{+} \mathbf{n}^{+}
$$

with $\mathbf{J}=\mathbf{R}^{T} \mathbf{J}^{R} \mathbf{R}$.

As a summary, the final equations to be solved read: 


$$
\begin{array}{r}
\sum_{\Omega_{l=1}}^{\Omega_{l=N e}} \int_{\Omega_{l}}\left[\mathbf{w} \cdot\left(\rho \frac{D \mathbf{u}}{D t}-\mathbf{b}\right)+\nabla \mathbf{w}::\left(\mu \nabla^{s} \mathbf{u}+p \mathbf{I}\right)\right] d \Omega-\int_{\Gamma_{\sigma}} \mathbf{w} \cdot \overline{\sigma_{n}} d \Gamma=0 \\
\sum_{\Omega_{l=1}}^{\Omega_{l=N e}} \int_{\Omega_{l}}\left[\mathbf{w}_{e} \cdot\left(\rho \frac{D \mathbf{u}}{D t}-\mathbf{b}\right)+\nabla \mathbf{w}_{e}::\left(\mu \nabla^{s} \mathbf{u}+p \mathbf{I}\right)\right] d \Omega-\int_{\Gamma_{\sigma}} \mathbf{w}_{e} \cdot \overline{\sigma_{n}} d \Gamma \ldots \\
\ldots-\int_{\Gamma_{\text {int }}}\left(\mathbf{w}_{e}^{+} \cdot\left(\mathbf{J}\|\mathbf{u}\|^{+}-p^{+} \mathbf{n}^{+}\right)+\mathbf{w}_{e}^{-} \cdot\left(\mathbf{J}\|\mathbf{u}\|^{-}-p^{-} \mathbf{n}^{-}\right)\right) d \Gamma-\int_{\Gamma_{l}} \mathbf{w}_{e} \cdot \sigma_{n}^{\text {neigh }} d \Gamma=0
\end{array}
$$

b) Mass conservation:

$$
\begin{array}{r}
\sum_{\Omega_{l=1}}^{\Omega_{l=N e}}\left[\int_{\Omega_{l}} w^{p} \nabla \cdot \mathbf{u} d \Omega-\int_{\Omega_{l}} \nabla w^{p} \cdot \mathbf{u}_{e} d \Omega\right]+\int_{\Gamma} w^{p} \cdot \mathbf{u}_{n e} d \Gamma=0 \\
\sum_{\Omega_{l=1}}^{\Omega_{l=N e}}\left[\int_{\Omega_{l}} w_{e}^{p} \nabla \cdot\left(\mathbf{u}+\mathbf{u}_{e}\right) d \Omega+\int_{\Gamma_{\mathrm{int}}}\left(w_{e}^{p+}\left\|\mathbf{u}_{e}\right\|^{+} \cdot \mathbf{n}^{+}+w_{e}^{p-}\left\|\mathbf{u}_{e}\right\|^{-} \cdot \mathbf{n}^{-}\right) d \Gamma \ldots\right. \\
\left.\ldots \int_{\Gamma_{l}} w_{e}^{p}\left(\mathbf{u}_{n e}^{\text {neigh }}-\mathbf{u}_{n e}^{\text {ele }}\right) d \Gamma\right]=0
\end{array}
$$$$
\text { It must be noted that in case that the integration by parts of the term }
$$
involved with the continuous weighting functions and the discontinuous enriched functions (second integral in the first line of (26)) is not performed, then the first line of (26) remains:

$\sum_{\Omega_{l=1}}^{\Omega_{l=N e}}\left[\int_{\Omega_{l}} w^{p} \nabla \cdot\left(\mathbf{u}+\mathbf{u}_{e}\right) d \Omega-\int_{\Gamma_{\mathrm{int}}} w^{p}\left\|\mathbf{u}_{e}\right\| \cdot \mathbf{n} d \Gamma+\int_{\Gamma_{l}} w^{p}\left(\mathbf{u}_{n e}^{\mathrm{neigh}}-\mathbf{u}_{n e}^{\mathrm{ele}}\right) d \Gamma\right]=0$

which means that without this integration by parts one integral must be added on the internal interfaces and along all the element boundaries enriched in order to preserve the mass conservation. 
In the previous equations, there are terms named $\sigma_{n}^{\text {neigh }}$ and $\mathbf{u}_{n}^{\text {neigh }}$ corresponding to the normal stress and normal velocity of the neighboring element where the integration is performed. In order to enable the condensation of the enriched degree of freedom at elemental level (EFEM) these two terms will be approximated with the corresponding stress and velocity in the integration element itself. In other words: $\sigma_{n}^{\text {neigh }}=\sigma_{n}^{\text {ele }}$ and $\mathbf{u}_{n e}^{\text {neigh }}=\mathbf{u}_{n e}^{\text {ele }}$.

With this approximation, the last integral on the element boundaries of the mass conservation equations becomes null remaining only the last integral on the element boundaries in the momentum equation. These integrals were named inter-element forces in [1] because they are similar to the introduction of a load on both boundaries of two neighboring elements. However, as explained in [1], the addition of these integrals must not be understood as the addition of a boundary load. It must be better interpreted as a $d o$ nothing boundary condition between the two neighboring elements. The do nothing boundary condition was first proposed in [26] to improve the outflow boundary condition in unbounded flows. It was later generalized to slip boundary condition in [27] and discussed also in [28]. In this new enriched space, the do nothing boundary condition will be used to improve the discontinuity existing between two elements in the case of elemental enrichment. As can be seen in the numerical examples, these inter-element forces improve considerably the accuracy of the elemental enrichment, decreasing (and in many cases eliminating) the artificial jump that appears between two neighbor elements due to the static condensation of the enriched DOF.

Unfortunately, in spite of using a Galerkin approximation, the interelement forces and the integration by parts of one of the terms in the mass conservation equation generate a non-symmetry stiffness matrix. Nevertheless, the improvements in the results that are obtained using this approximation counteract the disadvantage of having asymmetric matrices.

\section{The Finite Element with the Enriched Shape function}

The enriched space for reproducing a kink or a jump inside a 2D triangle may be obtained subdividing the element in three sub-elements and using the standard FE shape functions of each sub-element, as shown by Figure 2.

For the case of kinks + jumps the triangle is subdivided in the same way but duplicating the nodes at the internal interface. The procedure to obtain the final stiffness matrix of each element to be assembled in the global stiffness matrix may be followed in Ref [1]. 
The stiffness matrix of each sub-element is assembled in one super-element of 5 nodes (for kinks) or 7 nodes (for kinks+ jumps). The inter-element forces are added on all the element boundaries in which an internal interface is present. Finally the enriched DOF are eliminated by static condensation following a standard procedure.

In the case of three-dimensional finite elements, the internal interfaces are composed by planar facets, which do not conform to the element faces. Again, the element can then be split into two sub-regions. Two possible situations have to be considered, since the reconstructed interface can be either a triangular or a quadrangular facet. In the first case, the tetrahedron is subdivided in 4 sub-elements, and in the second case, is divided in 6 subelements. Then, the enriched DOF's are eliminated by static condensation as usual.
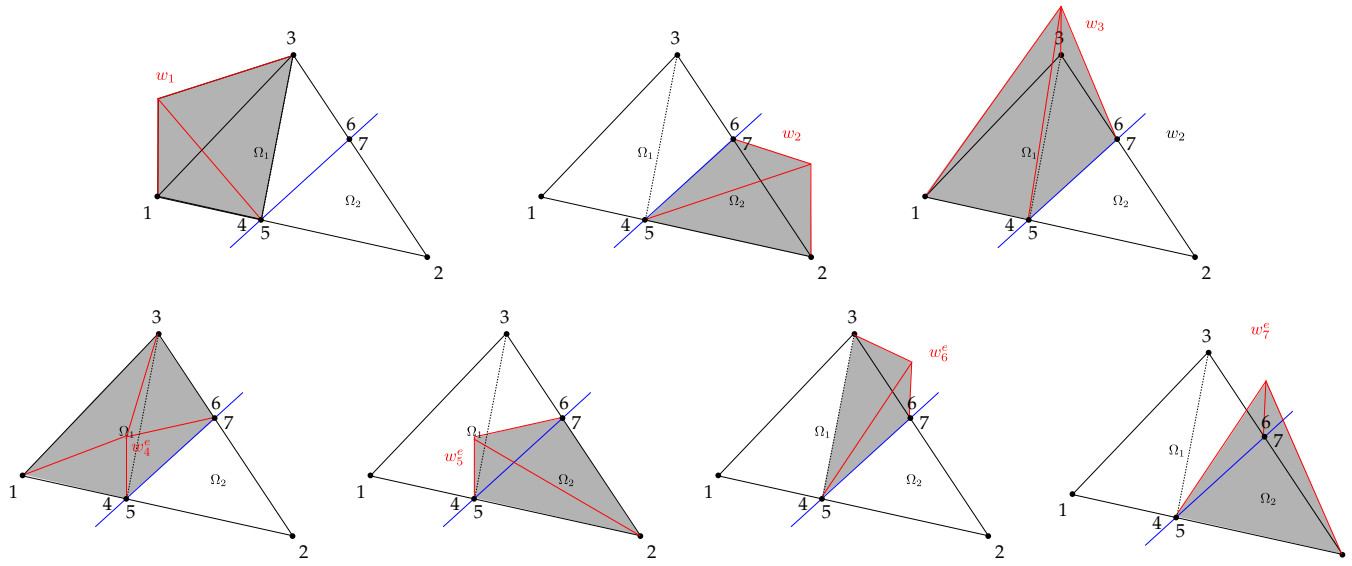

Figure 2: Standart and enriched shape functions for kink and jump. Two-dimensional case.

Two different cases of pathological problem have been referred in the previous paper and the same solution will be used now. One case is related to geometrical problems involved when the internal interface is near a node, very close to an interface or both. The other case is related to which decision must be taken when there are more than one result in the same position as currently occur in the elemental enriched space. The readers are referred to the previous paper in order to learn about the solution adopted for both cases.

Concerning the inertial terms $\int_{\Omega_{l}} \mathbf{w} \cdot \rho \frac{D \mathbf{u}}{D t} d \Omega$ the enrichment space was not considered, using for this terms the standard FE shape functions corre- 
sponding to the linear triangle or lineal tetrahedral respectively. For the case of an Eulerian formulation the term $\frac{D \mathbf{u}}{D t}$ was replaced by $\frac{\partial \mathbf{u}}{\partial t}+\mathbf{u} \cdot \nabla \mathbf{u}$ with a standard SUPG stabilized scheme [25] without any enriched space was used to avoid spurious oscillation due to the convective terms.

It must be also noted that in the case of equal-order velocity-pressure elements as those used in the examples presented next, the conservation of mass equation must also be stabilized. A standard PSPG [25] stabilization was used here without enrichment in its functional space.

\section{Numerical examples}

The numerical examples chosen in this section are fluid mechanics problems where the unknown functions are the velocity and the pressure fields. The main objective is to highlight the possibilities of the EFEM for these kinds of vectorial solutions and put in evidence the accuracy of the elemental enrichment in this context. To see the errors compared with analytical solutions and the convergence of the method for more academic cases, the readers are invited to see the Reference [1].

\subsection{Couette flow with two fluids}

The first case analyzed is the incompressible flow counterpart of the cases named one-dimensional kink and one-dimensional jump presented in the previous work of the authors [1]. Instead of solving thermal problems, in the current case the unknowns are the velocity and the pressure fields. According to the geometry and boundary conditions presented in Figure 3, the test represents the problem of two plane plates with different tangential velocities between them, also known as Couette Flow. If the same fluid at each side of the interface is considered and $J_{\tau}=J_{n}=\infty$ is imposed, the solution is the classical linear velocity profile. In the case of fluids with different viscosities, a kink of the velocity is produced on the interface due to a discontinuity in their gradients. The analytical solution presented in (28), while the pressure is constant over all the domain, taking the same value imposed at the inlet.

$$
\mathbf{u}_{x}(x, y)=\left\{\begin{array}{cc}
\frac{2 \mu_{2}}{\mu_{1}+\mu_{2}} y & y \leq 0.5 \\
1-\frac{2 \mu_{1}}{\mu_{1}+\mu_{2}}(1-y) & y>0.5
\end{array}\right. \text {. }
$$



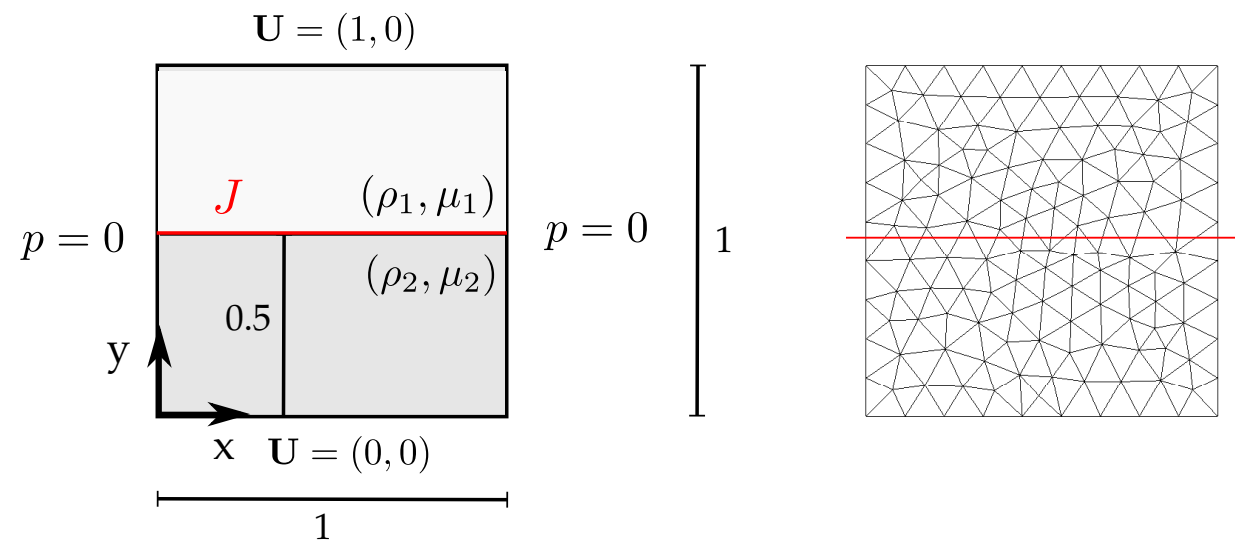

Figure 3: Couette flow case configuration and unstructured mesh employed. Units are $\mathrm{m} / \mathrm{s}$ for velocity, $\mathrm{Pa}$ for pressure, Pas for viscosity and $\mathrm{kg} / \mathrm{m}^{3}$ for density.

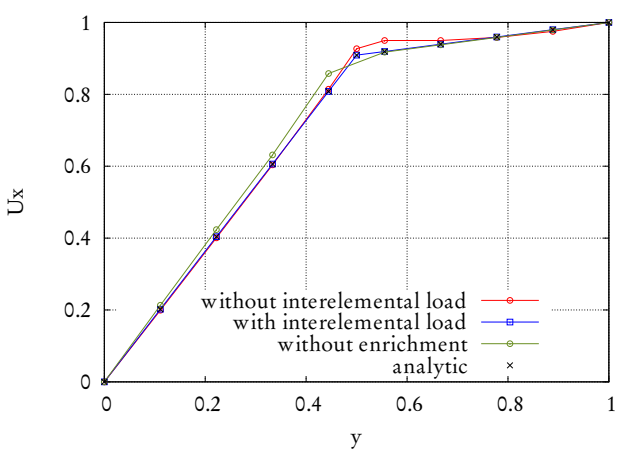

(a) $x=1$ slice

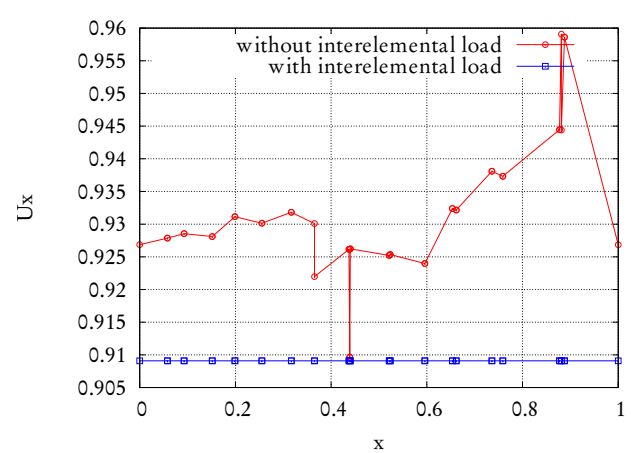

(b) $y=0.5$ slice

Figure 4: Couette flow with kink generated by viscosity jump. Horizontal velocity over horizontal and vertical slices.

The first test considers a viscosity jump $\mu_{1}=1, \mu_{2}=10$ with constant density $\rho_{1}=\rho_{2}=1$ and continuity of the solution at interface, i.e. $J_{\tau}=J_{n}=\infty$. Figure 4a presents the analytic solution over a slice at $x=1$ compared with three different numerical solutions obtained employing the unstructured mesh showed in 3. The solution with standard FEM, i.e. without enrichment, fails capturing the kink and estimates wrongly the velocity gradient which results in an unacceptable solution even in this simple case. As expected, using enrichment improves the kink capturing. However, as discussed before in this work, the lack of the inter elemental load term leads to a solution which has some deficiencies specially in the region of small vis- 
cosity. That variational crime is clearly solved incorporating the mentioned term in the elemental assemble (solution with inter elemental load). This difference is highlighted by Figure $4 \mathrm{~b}$ where the value of $\mathrm{x}$-velocity over the enriched degrees of freedom over the interface is presented. It is noticeable how including the inter elemental load the solution obtained matches the analytic one while not employing it the solution is poor. Moreover, some enriched nodes at same physical point have different velocity values depending on the interface side where they are. As seen in this first case, the only numerical strategy which guarantees an accurate solution when the mesh does not match the interface is employing enrichment with interelemental load.

The second example considers a jump in the unknowns. A jump of the velocity in an incompressible flow problem may be considered when there are two fluids in contact but supposing that there is a material in between the two domains which imposes some restriction for momentum transference. It is, for instance, how acts the surface tension at the interface of two fluids, or the presence of a plate between two fluids with same or different physical properties. The amount of momentum transfer for each direction, i.e. tangential and normal to the interface, is regulated by the coefficient of the matrix $\mathbf{J}$ described in (22). Although $J_{n}$ can take any value depending on the problem, in this case and the following ones in this work, we will always consider impenetrability at the interface, i.e. imposing the same normal velocity at both sides of the interface with $J_{n}=\infty$. Cases with $J_{n} \neq \infty$ are not treated in this work, but could be useful in the case of curved interfaces where a slipping condition should be insured, but as the interface is represented by straight lines a locking of the flow is found at interface. Therefore, in order to simplify the notation, we will use $J$ when we refer to $J_{\tau}$.

Figure 5 compares the solutions in a problem with same fluid at both sides of the interface $\left(\mu_{1}=\mu_{2}=\rho_{1}=\rho_{2}=1\right)$ when $J$ varies. In all the cases, the enrichment proposed with the inter-elemental forces gives the exact result in any horizontal line.

Remark 1. Is important to mention that the interelemental load evaluation on the edges where $\Gamma_{l} \cap \Gamma_{\sigma} \neq \emptyset$ must consider $\sigma_{n}^{\text {neigh }}=\bar{\sigma}_{n}$, i.e. the traction is dictated by the boundary condition. On the other hand, for boundaries where $\Gamma_{l} \cap \Gamma_{\mathbf{u}} \neq \emptyset$, an approximation employing $\sigma_{n}^{\text {neigh }}=\sigma_{n}^{\text {ele }}$ is adopted as in the internal edges.

Remark 2. The condensation of new degrees of freedom introduces nonlinearities which must be solved iteratively. Therefore, previous iteration values for enriched nodes must be stored. During the first non-linear iteration 


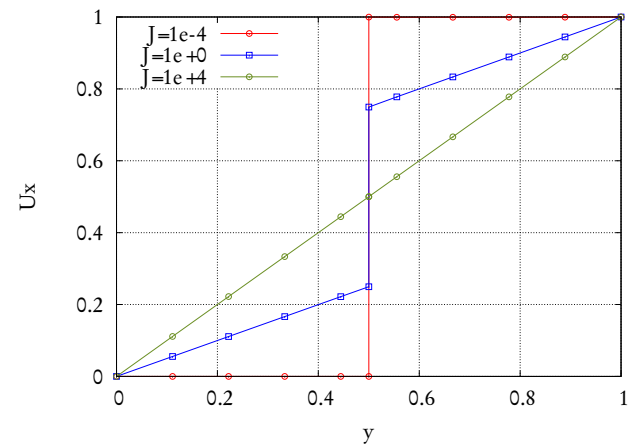

Figure 5: Couette flow with a jump. Solutions for different values of $J$.

a linear interpolation among parent nodes could be considered to impose an initial value on enriched nodes.

\subsection{Moving flap valve}

Couette case shows the capability of the enriched space proposed to improve the accuracy of the solution when a coarse mesh does not match the interface and also there is a kink or/and a jump of the unknowns. However, if the interface position is fixed, a matching mesh (with duplicated nodes at the interface) can be used or strategies as XFEM can be applied and the accuracy of the results will be at least equal.

In this context, this case proposes a moving interface where a matching mesh strategy would require remeshing every time-step, or the matrix of the equation system of XFEM techniques should be resized according to the variation of positioning and number of extra degrees of freedom. This task requires extensive computational time which can be avoided if the EFEM proposal of this work is employed, where exactly the same matrix graph is used, this means that the solution matrix has exactly the same DOFs although the interface position may be continnuously moving.

Figure 6 shows a two-dimensional homogeneous and incompressible flow problem which represents a pipe with a flap valve characterized by a moving interface. The valve position is fixed at the inlet and a rigid oscillating movement is imposed following the equation $y(x, t)=0.5+x 0.15 \sin (2 \pi t / T)$. Imposing impenetrability, $\left.\mathbf{u}_{n}\right|_{\Gamma_{i n t}}=0$ and discontinuity of tangential velocities over it, i.e. $J=0$, the interface models a solid and slip valve. The flow rate imposed at inlet is $1 \mathrm{~m}^{3} / \mathrm{s}$ and it must be kept constant at outlet, this condition will accelerate the flow in the region where the valve constrains its 
area.

Background fixed mesh employed consists in 36 by 18 structured nodes conforming 1296 triangles. The oscillation period is $T=10^{5}$ while the timestep employed is $\Delta t=5 \times 10^{3}$. These huge temporal steps are selected in order to avoid the influence of the mass matrix over the system. In this context, each time-step is treated as a pseudo-stationary state.

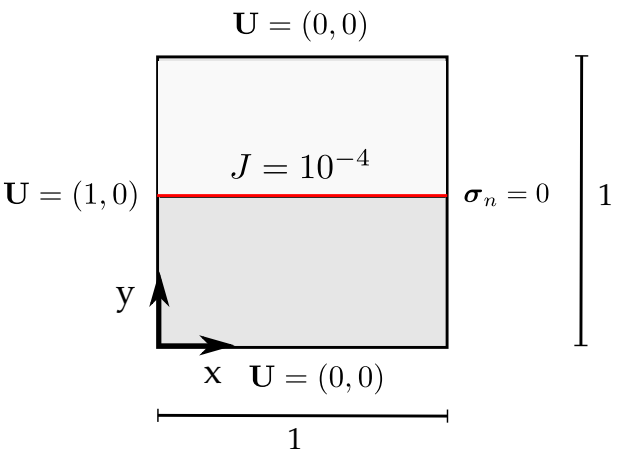

Figure 6: Geometry and boundary condition for flap valve case. Red line represents the valve position at $t=0$

Figure 7 shows the magnitude of the velocity at different valve positions. As a validation of the results, the difference between the inlet flow rate and the outlet flow rate is considered. Maximum differences are about of $1 \%$ and could be attributed to the coarse background mesh employed. This fact can be observed in the comparison among the velocity profile at outlet shown in Figure 8. When the valve is centered (for example, at starting position) the solution is the classical parabolic profile with maximum $|\mathbf{u}|_{\text {max }}=\frac{3}{2}|\mathbf{u}|_{\text {inlet }}$. Solutions for other stages present a jump at the interface, and the velocity varies its maximum according to the contraction or expansion of the region transversal area, in order to guarantee conservativeness.

Remark 3. In this case, a slip condition is employed over the interface. A possible improvement could be including the modeling of a boundary layer through a wall law, adjusting the value of $J$.

\subsection{Elbow with internal wall}

The basis of this case are similar to the previous one. The flow inside a two-dimensional pipe with a valve is also calculated. However in this case the pipe geometry presents a 90 degrees curve conforming an elbow. This 


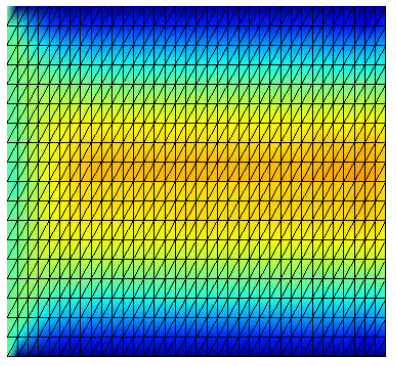

(a) $t^{*}=0$

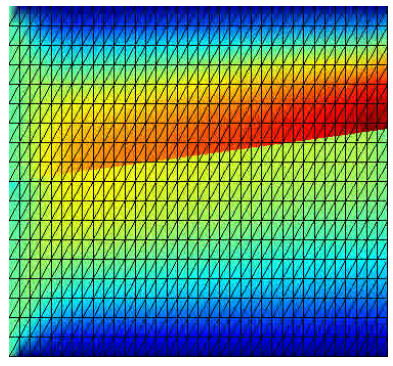

(b) $t^{*}=1 / 4$

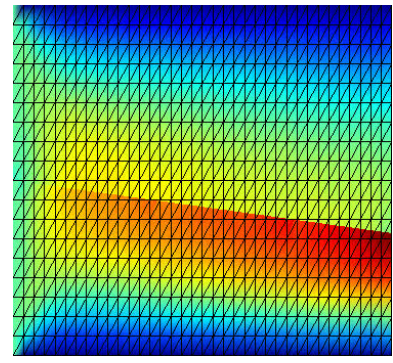

(c) $t^{*}=3 / 4$

Figure 7: Moving valve case. $|\mathbf{u}|$ at several snapshots. Scale from $|\mathbf{u}|=0 \mathrm{~m} / \mathrm{s}$ (blue) to $|\mathbf{u}|=2.2 \mathrm{~m} / \mathrm{s}(\mathrm{red})$.

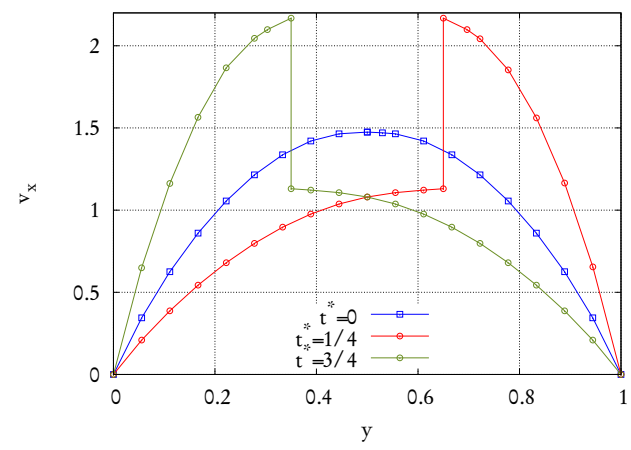

Figure 8: Moving valve case. Velocity profile at outlet $(x=1)$ for different valve positions.

more complex case is employed to validate the quality of the elemental approximations used when the interface is not a straight line.

With the aim of reproducing the procedure on general simulations, instead of using the analytical expression, a distance function field $\psi(\mathbf{x})$ which has values over the mesh nodes is employed to determinate the interface position. Using the standard linear shape functions, an interface element estimates the interface position as the straight line which accomplishes $\psi(\mathbf{x})=0$. More details about this standard algorithm can be found in [29]. This procedure makes that, over a curve, the interface normals $\mathbf{n}$ varies element by element. This fact could introduce discrepancies of the unknowns values computed by the pair of enriched degrees of freedom at the same position but on different elements.

Figure 9 presents the geometry and boundary conditions employed. Note that the radius of the duct is not constant, then the interface position reduces the transversal area of the lower region after the curve. A Cartesian grid with 
2158 elements is employed. A one-phase flow is considered with viscosity and density of unity. Taking as reference length the radius of the duct, the Reynolds number simulated is $R e=1$.

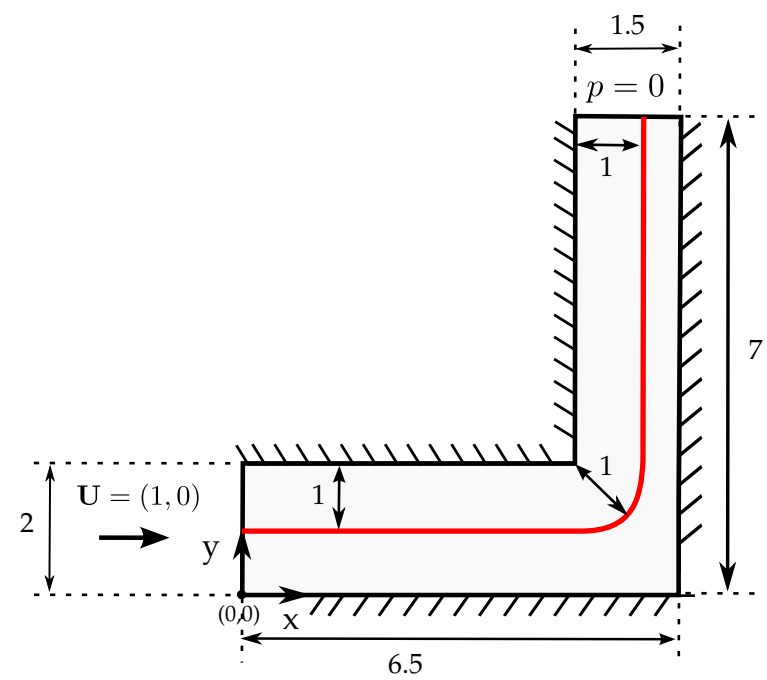

Figure 9: Elbow case. Geometry and boundary conditions. Red line represents the interface position.

Pressure and velocity solutions are shown in Figure 10. The contraction in the right region of the pipe after the curve generates acceleration of the fluid in order to keep the total flow constant. In this case, differences between inlet and outlet flows are about of $4 \%$. The error is due to a small penetrability of the interface because of the procedure for computing the normals, and others as the use of a too coarse mesh, which accelerates also the flow of the left region after the curve, even the duct radius is kept constant. The pressure field also presents a jump along the interface, which is expected due to the different driving forces required by each region in order to satisfy the inlet flow imposed.

\subsection{The flow through a moving sail of a sailboat}

An interesting application case of the enrichment space proposed is presented here where the flow around a sailboat is simulated. The sail, an impenetrable, thick, and deformable material is modeled as an interfase with discontinuity in both normal and tangential velocities, which implies that the flow at one side does not interact directly with the flow at the other side of the sail. 


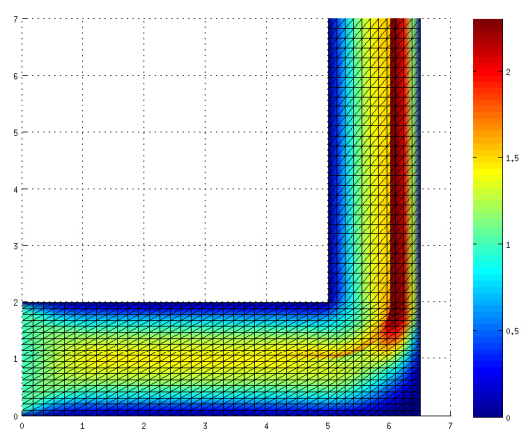

(a) velocity magnitude

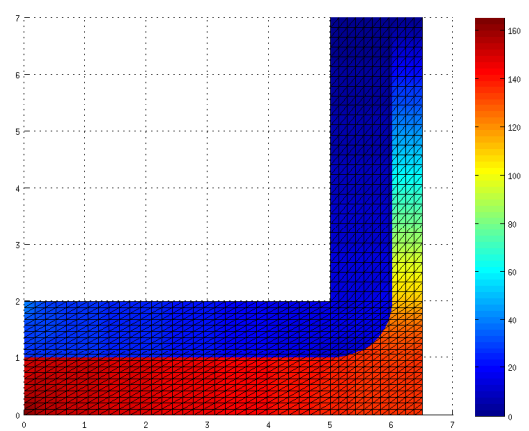

(b) pressure

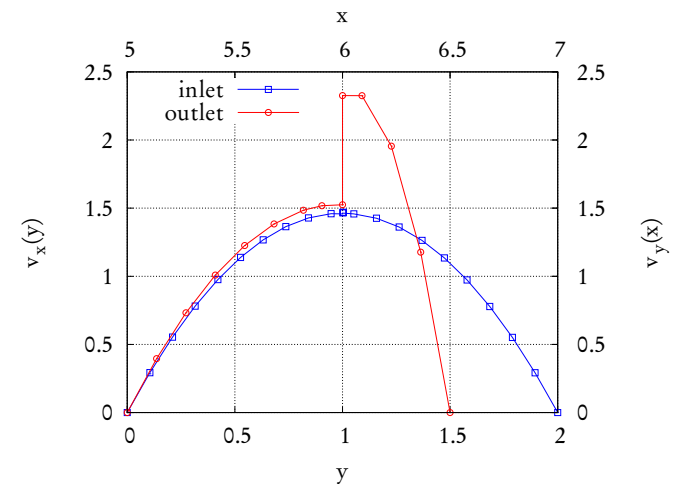

(c) Comparison of velocity profile at inlet and outlet.

Figure 10: Elbow case. Solutions and slices. 
Sail position is represented by the analytic function $\psi(x, t)=a x^{2}+b x+c$ with $\psi(0.3)=0.347, \psi(0.7)=0.707$ and $c=\frac{3}{2} \sin (2 \pi t / T)$. An uniform Cartesian grid of 75 by 25 elements subdivided into triangles is employed. A one-phase flow is considered being its properties $\mu=1$ and $\rho=1$. Therefore $R e=1$ taking as reference length the channel width. In order to model that the interface begins and ends inside the domain, the jump coefficient follows:

$$
J=\left\{\begin{array}{cc}
0 & 0.3 \leq x \leq 0.7 \\
\infty & \text { otherwise }
\end{array} .\right.
$$

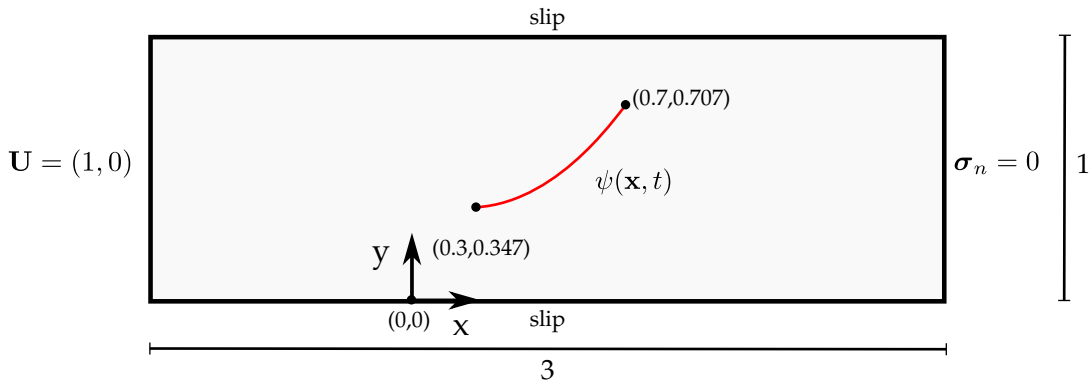

Figure 11: Geometry and boundary condition for sail case. Red line represents the sail position at $t=0$

Solution is presented in Figure 12. SubFigures 12a and 12b show the magnitude of the velocity and the pressure fields, with arrows representing the direction of the flow, respectively. Although the Reynolds number of the problem is low then the flow does not produce shedding, the velocity and pressure fields have the expected features. Pressure has maximum over the side of impact of the sail, while the minimum occurs behind the sail where flow detachment is observed. Flow surrounds properly the shape without permeabilities. The snapshots shown in Figures 12c to 12h, which present the behavior of the flow for the different position of the sail, also accomplishes the mentioned features.

\subsection{External and internal fluid flow around a droplet}

In this case an internal circulation pattern developed in a heavier fluid (as water) droplet due to the movement of the surrounding moving lighter fluid (as air) is solved. Analyzing the drop's behavior and its interaction with the environment is important, for example, for spray technology physics, 


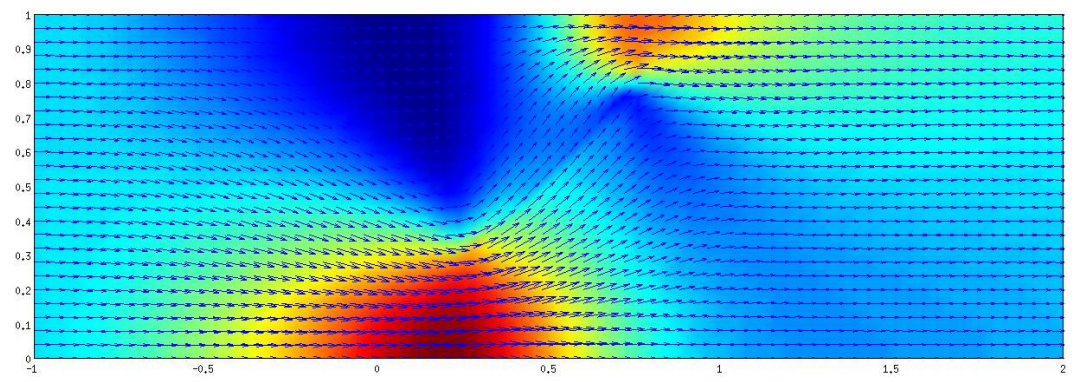

(a) $|\mathbf{u}|$ at $t=5000 \mathrm{~s}$

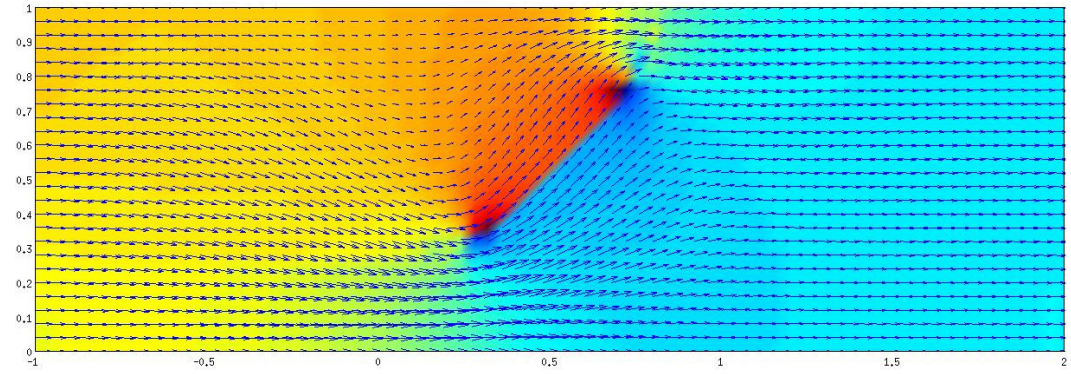

(b) pressure at $t=5000 \mathrm{~s}$
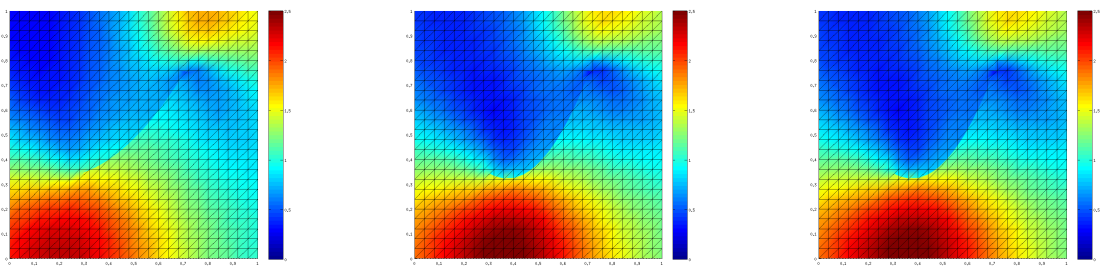

(c) $|\mathbf{u}|$ at $t=10000 \mathrm{~s}$

(d) $|\mathbf{u}|$ at $t=20000 \mathrm{~s}$

(e) $|\mathbf{u}|$ at $t=40000 \mathrm{~s}$
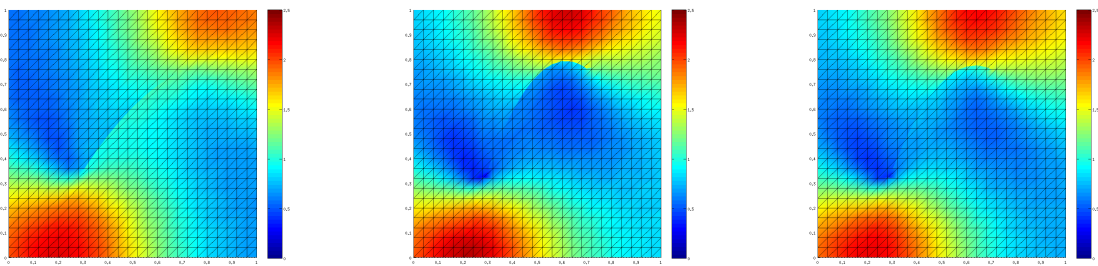

(f) $|\mathbf{u}|$ at $t=60000 \mathrm{~s}$

(g) $|\mathbf{u}|$ at $t=75000 \mathrm{~s}$

(h) $|\mathbf{u}|$ at $t=90000 \mathrm{~s}$

Figure 12: Moving sail case. Solution fields at several times. 
injection in combustion chambers, etc. and its understanding is required to properly model those applications.

Case configuration (geometry, boundary and initial conditions) are presented in Figure 13. The condition of $\left.\mathbf{u}_{n}\right|_{\Gamma_{i n t}}=0$ is imposed at the interface, but two different cases are solved selecting different values for $J$. Physical parameters employed are $\rho=1, \mu_{1}=1, \mu_{2}$ is variable and surface tension is not modeled. Defining $R e=\frac{\rho|\mathbf{u}| a}{\mu}$, being $a=0.15$ the droplet radius, the Reynolds number simulated is $R e=0.15$ The mesh employed consists in a Cartesian grid of 60 by 30 cells split into triangles.

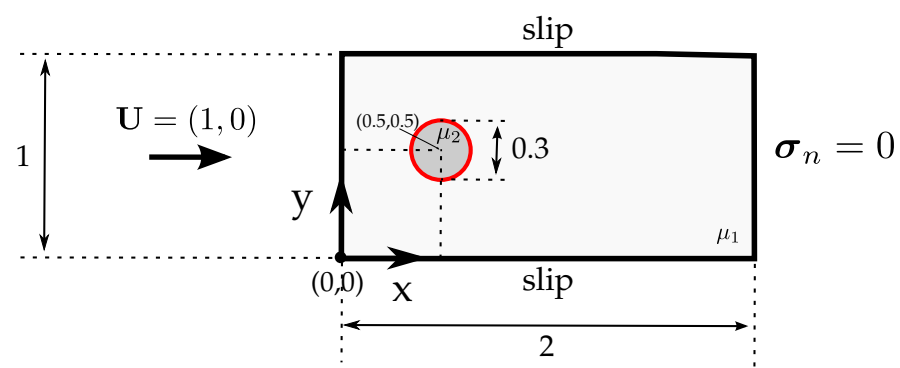

Figure 13: Fluid drop case. Geometry and boundary condition. Red line represents the interface position at $t=0$ where impenetrability is imposed

The first case imposes $J=0$, i.e. discontinuity in the tangential velocity at the interface, and $\mu_{2}=0.01$. Its solution should not induce almost any flow inside the drop even though the large viscosity difference. This case is inspired on a fluid flow surrounding a solid sphere or a fluid droplet isolated by a rigid membrane. Figures $14 \mathrm{a}$ and $14 \mathrm{~b}$ show the horizontal and vertical components of the velocity respectively for the solution at time $t=0$. Note that inside the drop both component vanishes and the exterior flow contours the shape. In Figure 17a the magnitude of velocity and base mesh employed are shown. It is noticeable how the velocity jump is captured even if the interface cuts an element.

On the other hand, the case with $J=\infty$ and $\mu_{2}=1$ allows momentum transfer along the interface inducing a flow inside the drop. Solution presented in Figures 15 and 17b show that the external fluid motion, in the horizontal direction, results in a doughnut shaped, toroid, flow within the drop known as a Hill's vortex. The cause of the internal circulation is the shear force at the drop surface created by the fluid moving along the surface and allowed by the $J$ selected. 


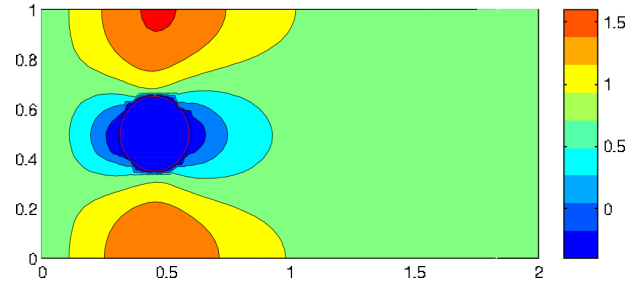

(a) $\mathbf{u}_{x}$ and isocontours

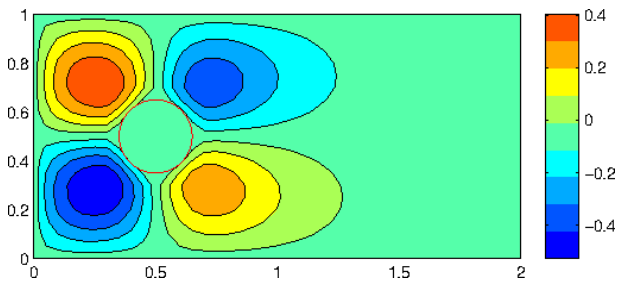

(b) $\mathbf{u}_{y}$ and isocontours

Figure 14: Fluid drop case with $J=0$.

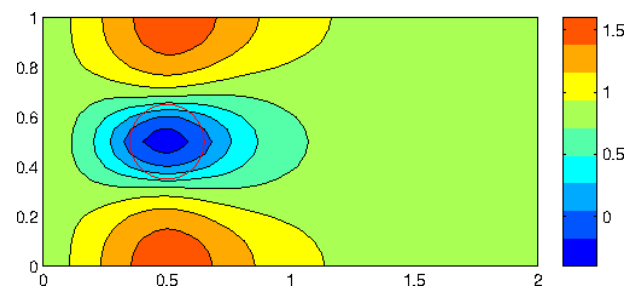

(a) $\mathbf{u}_{x}$ and isocontours

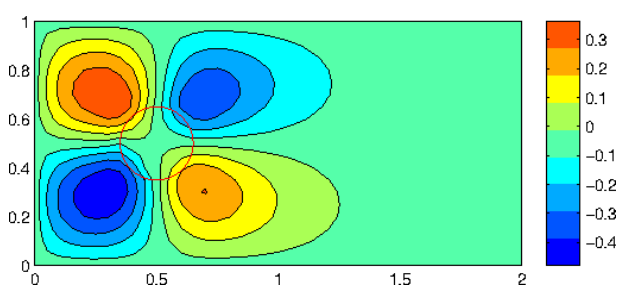

(b) $\mathbf{u}_{y}$ and isocontours

Figure 15: Fluid drop case with $J=\infty$.

A comparison between the solutions at different $J$ is presented by Figure 16. Velocity profiles along horizontal $(x=0.5)$ and vertical $(y=0.5)$ axis show clearly as the velocity vanishes inside the drop in the case of $J=0$. In the solution for $J=\infty$ some check-points can be analyzed which guarantee a physical solution: considering creeping flow the velocity magnitude at the interface must be $|\mathbf{u}|=|\mathbf{u}|_{\text {inlet }} / 2.0$ which is well accomplished by solution, particularly this can be proven from Figure 16 at the impact point where $\mathbf{u}_{x}(0.35,0)=0$ and at the higher and lower points of the drop $\mathbf{u}_{x}(0.5,0.65)=$ $\mathbf{u}_{x}(0.5,0.35)=0.5$. Both velocity profiles inside the drop describe a parabola with similar minimum value, showing the presence of the typical pair of vortices induced by an external moving fluid.

The enriched space employed allows also to capture the discontinuity of the pressure field at the interface. In the case of $J=\infty$ the maximum pressure due to the flow impact to the shape occurs in the same numerical point than the minimum inside the drop, while the jump at the other side of the drop is smaller. The captured pressure jumps is also noticeable when $J=0$ is employed. In the latter, the pressure keeps constant to the reference value imposed in one of the droplet nodes $(p=0)$. 


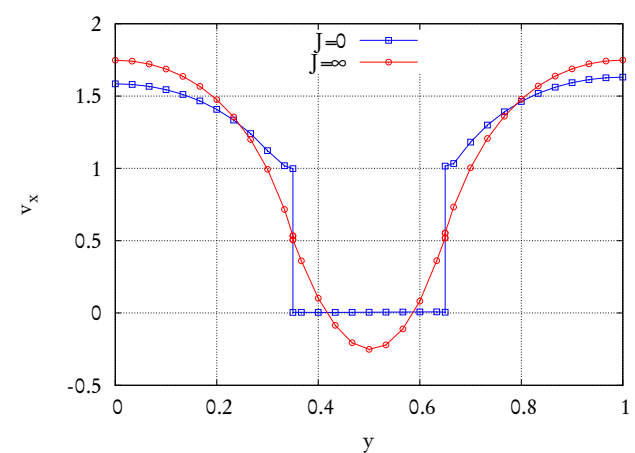

(a) $\mathrm{x}$-velocity along the line $x=0.5$

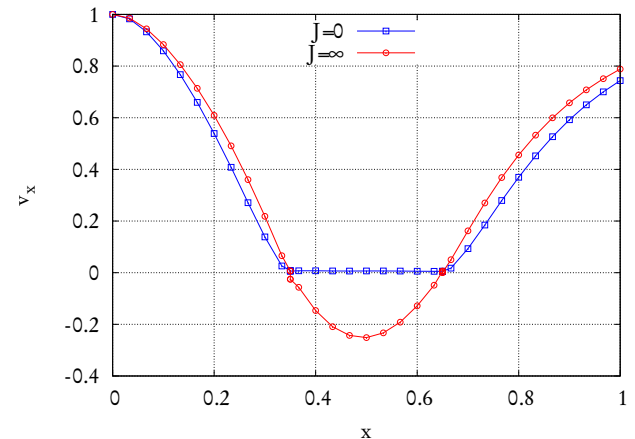

(b) $\mathrm{x}$-velocity along the line $y=0.5$

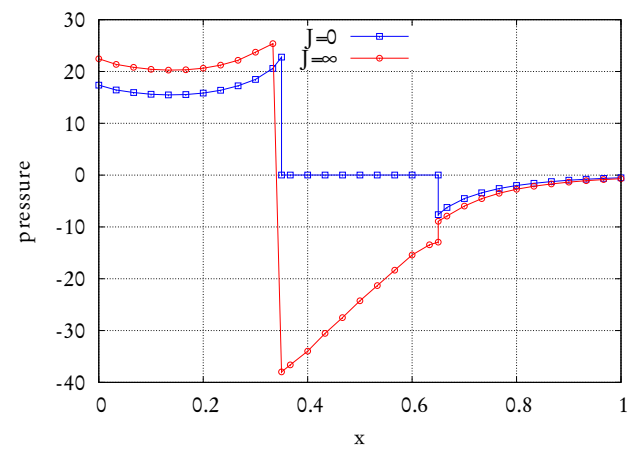

(c) pressure along the line $y=0.5$.

Figure 16: Fluid drop case. Profiles of velocity and pressure with different values of $J$.

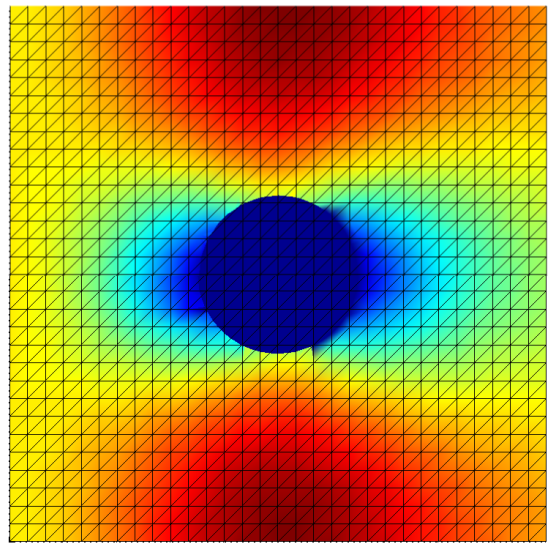

(a) Mesh and $|\mathbf{u}|$. Scale goes from blue to red (0.0 to 1.6). Case $J=0$

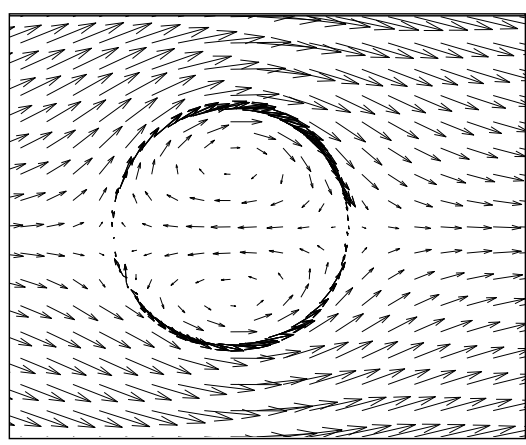

(b) Vectorial representation of velocity field. Case $J=\infty$.

Figure 17: Fluid drop case. Velocity fields with different values of $J$. 


\section{Conclusions}

This paper describes how to extend the ideas of using an enriched functional space to capture the discontinuities normally present at interfaces of multifluid flows, either kinks or jumps. After being successfully applied to thermal problems, in this paper the theory was adapted for a vectorial non linear momentum equation constrained by the incompressibility condition like in fluid mechanics. Instead of using very refined mesh to capture this flow features, this methodology save a lot of DOFs using a special defined functional space that allows for representing in a synthetic way discontinuities in either the function itself or its gradients. Moreover, for problems where the interfaces are constantly moving all around the domain, this strategy based on elemental enrichment (EFEM) may be more adequate than XFEM in terms of efficiency. But, similar to the thermal case, the inter elemental loads should be included in order to diminish the variational crime produced by EFEM when using a linear representation along the interface for both, the kinks or the jumps. Finally, this new method to capture the discontinuities at the interface opens a new horizon in terms of modeling the surface tension and the wall law for turbulence modeling avoiding a very detailed mesh around the interface.

\section{Acknowledgements}

The research leading to these results has received funding from the European Research Council under the European Union's Seventh Framework Programme (FP/2007-2013) / ERC Grant Agreement n. 320815 (ERC Advanced Grant Project "Advanced tools for computational design of engineering materials" COMP-DES-MAT). The authors also wish to express their gratitude to Professor Xavier Oliver for his kind and interesting answers to several discussions shared at CIMNE. The authors also wish to offer their thanks to the CONICET, the Universidad Nacional del Litoral, and the ANPCyT for their financial supports through Grants PIP-2012 GI 11220110100331, CAI+D 201150120110100435 LI, and PICT-2013 0830.

\section{References}

[1] S. R. Idelsohn, J. M. Gimenez, J. Marti, N. M. Nigro, Elemental enriched spaces for the treatment of weak and strong discontinuous fields, 
Computer Methods in Applied Mechanics and Engineering 313 (2017) $535-559$.

[2] S. Unverdi, G. Tryggvason, A front-tracking method for viscous, incompressible, multi-fluid flows, Journal of Computational Physics 100 (25-37) 1992.

[3] D. Gueyffier, J. Lie, A. Nadim, R. Scardovelli, S. Zaleski, Volumeof-fluid interface tracking with smoothed surface stress methods for three-dimensional flows., Journal of Computational Physics 152 (1999) 423-456.

[4] S. Popinet, S. Zaleski, A front-tracking algorithm for accurate representation of surface tension., International Journal for Numerical Methods in Fluids 30 (1999) 775-793.

[5] C. Hirt, B. Nichols, Volume of fluid (vof) method for the dynamics of free boundaries, J. Comput. Phys 39(1) (1981) 201-225.

[6] D. Kothe, W. Rider, S. Mosso, J. Brock, J. Hochstein, Volume tracking of interfaces having surface tension in two and three dimensions., Technical Report Technical Report AIAA 96-0859, AIAA, 1996.

[7] S. Cummins, M. Francois, D. Kothe, Estimating curvature from volume fraction, Computers and Structures 83 (2005) 425-434.

[8] D. Adalsteinsson, J. Sethian, A fast level set method for propagating interfaces, Journal of Computational Physics 118 (1995) 269-277.

[9] J. Sethian, Evolution, implementation, and application of level set and fast marching methods for advancing fronts., Journal of Computational Physics 169 (2001) 503-555.

[10] S. Osher, R. Fedkiw, Level set methods: an overview and some recent results, Journal of Computational Physics 169 (2001) 463 - 502.

[11] J. Guermond, L. Quartapelle, A projection fem for variable density incompressible flows, Journal of Computational Physics 165 (2000) 167188. 
[12] S. Idelsohn, N. Nigro, J. Gimenez, R. Rossi, J. Marti., A fast and accurate method to solve the incompressible navier-stokes equations, Engineering Computations 30-Iss:2 (2013) 197-222.

[13] S. Idelsohn, J. Marti, P. Becker, E. Oñate, Analysis of multifluid flows with large time steps using the particle finite element method, International Journal for Numerical Methods in Fluids 75 (2014) 621-644.

[14] J. Gimenez, L. González, An extended validation of the last generation of particle finite element method for free surface flows, Journal of Computational Physics 284 (2015) 186 - 205.

[15] J. Gimenez, Enlarging time-steps for solving one- and two-phase flows with the Particle Finite Element Method, Ph.D. thesis, Facultad de Ingeniería y Ciencias Hídricas (FICH) Centro de Investigacion de Métodos Computacionales (CIMEC) Universidad Nacional del Litoral, 2015.

[16] S. Ganesan, G. Matthies, L. Tobiska, On spurious velocities in incompressible flow problems with interfaces, Computer Methods in Applied Mechanics and Engineering 196 (2007) 1193-1202.

[17] P. D. Minev, T. Chen, K. Nandakumar, A finite element technique for multifluid incompressible flow using eulerian grids, Journal of Computational Physics 187 (2003) 255-273.

[18] J. Chessa, T. Belytschko, An extended finite element method for twophase fluids., Journal of Applied Mechanics 70 (2003) 10-17.

[19] T. Strouboulis, I. Babuška, K. Copps, The design and analysis of the generalized finite element method., Computer Methods in Applied Mechanics and Engineering 181 (2000) 43-69.

[20] S. Gross, A. Reusken, An extended pressure finite element space for two-phase incompressible flows with surface tension., Journal of Computational Physics 224 (2007) 40-58.

[21] J. W. Barrett, H. Garcke, R. Nürnberg, A stable parametric finite element discretization of two-phase navier-stokes flow, Journal of Scientific Computing 63 (2015) 78-117. 
[22] J. Oliver, A. Huespe, E. Samaniego, A study on finite elements for capturing strong discontinuities., Int. J. Num. Meth. Engrg. 56 (2003) 2135-2161.

[23] J. Oliver, A. Huespe, P. Sánchez, A comparative study on finite elements for capturing strong discontinuities: e-fem vs x-fem., Comput. Methods Appl. Mech. Engrg. 195 (2006) 4732-4752.

[24] R. Ausas, G. Buscaglia, S. Idelsohn, A new enrichment space for the treatment of discontinuous pressures in multi-fluid flows, International Journal for Numerical Methods in Fluids 70 (2012) 829-850.

[25] T. Tezduyar, Stabilized finite element formulations for incompressible flow computations, Advances in Applied Mechanics 28 (1991) 1-44.

[26] T. Papanastasiou, N. Malamataris, K. Ellwood, A new outflow boundary condition, International Journal for Numerical Methods in Fluids 14 (1992) 587-608.

[27] M. Behr, On the application of slip boundary conditions on curved boundaries., International Journal for Numerical Methods in Fluids 45 (2004) 43-51.

[28] H. Coppola-Owen, R. Codina, A free surface finite element model for low froude number mould filling problems on fixed meshes, Int. J. Numer. Meth. Fluids 66 (2011) 833-851.

[29] J. Gimenez, L. González, An extended validation of the last generation of particle finite element method for free surface flows, Journal of Computational Physics 284 (2015) 186 - 205. 